\title{
A Hybrid Scheme for the Near Wall Treatment of Building Cube Method*
}

\author{
Xinrong SU ${ }^{* *}$, Daisuke SASAKI** and Kazuhiro NAKAHASHI** \\ ** Department of Aerospace Engineering, Tohoku Univeristy \\ 6-6-01 Aoba, Aoba-ku, Sendai-shi, Miyagi, Japan \\ E-mail: naka@ad.mech.tohoku.ac.jp
}

\begin{abstract}
In this paper we explore a new hybrid method for the near wall treatment of viscous computation with block structured Cartesian mesh. Cloud of points are generated to resolve the near wall boundary layer and most of the points are distributed on onedimensional lines along the wall normal directions, thus enabling the usage of high order scheme for the wall-normal flow derivative. In streamwise direction the flow derivative is approximated using second order least-square meshless method. Compared to the existing wall treatment methods for block structured Cartesian mesh, the current approach retains the flexibility of the least-square method and also offers the opportunity of high order accuracy along the wall-normal direction. A simple method is proposed to generate the near-wall points and it relieves the burden of body-fitted near-wall mesh generation. The current approach is tested with several high Reynolds number flows and the results are discussed.
\end{abstract}

Key words : Compressible Flow, Turbulent Flow, Numerical Simulation, Boundary Condition, Finite Difference Method

\section{Introduction}

It is well known Computational Fluid Dynamics (CFD) offers the ability to perform highfidelity predictions of the full field vehicle flows at low cost compared to wind tunnel tests. While the accurate and fast turn-around simulation around complex geometry still demands new algorithms and meshing tools. Of all the difficulties, the computation time related to the process from $\mathrm{CAD}$ to mesh generation increases dramatically relative to the time needed for the solution of governing equations on the modern high performance computers.

Of all the mesh types, multi-block structured mesh is body-fitted and offers the capability of implementing highly accurate methods, such as high order WENO scheme ${ }^{(1)}$ and the compact finite difference ${ }^{(2)}$. For complex geometry, the generation of the multi-block topology and the high quality mesh still needs lots of labor work and thus lacks automation. On the other hand, unstructured mesh provides considerable flexibility for complex geometry and the mesh generation process can be automated with less manual interactions. For unstructured mesh, currently second order finite volume method is mostly used and it still needs an expert spend several days to generate the mesh for complex configurations.

Cartesian grids, especially block structured Cartesian mesh are simple to generate and to adapt according to the local flow features. The block based structured mesh inherently supports the use of high order schemes. The generation of Cartesian mesh can be made fully automatic and robust for complex configurations. The Building Cube Method (BCM) has been proposed by Nakahashi et al. ${ }^{(3),(4)}$. In the BCM method, every block is composed of structured Cartesian mesh and is denoted as a cube and every cube has the same number of isotropic mesh points. In the near wall region the flow is locally anisotropic and the adaption of the mesh size to the local boundary layer length scale would result in huge amount of mesh

*Received 25 Jan., 2012 (No. 12-0037) [DOI: 10.1299/jfst.7.197]

Copyright (c) 2012 by JSME 
points. For turbulent flows, the boundary layer thickness can be estimated by:

$$
d=0.37 R e^{-0.2}
$$

For example at $R e=10^{6}$, in order to fully resolve the boundary layer, the number of total mesh points would exceed $10^{10}$. Thus the use of block-structured Cartesian mesh for high Reynolds number flows is beyond the current capability.

In order to use the BCM method for high Reynolds number flows, Ishida et al. ${ }^{(5)}$ proposed to couple the BCM method in the off-wall region with the least-square meshless method in the near-wall region. Munikrishna and Balakrishnan ${ }^{(6)}$, also Jahangirian and Hashemi ${ }^{(7)}$ pro- $^{-}$ posed to use the second order least-square method for the near-wall treatment of unstructured Cartesian mesh. As the name implies, meshless method does not need the topology information, such as the node-to-node, face-to-cell. Instead, clouds of supporting points are used to approximate the flow derivatives. The least-square meshless method offers extraordinary flexibility for the near wall treatment. Currently the widely used least-square method is at most second order accurate, in theory it is possible to design arbitrary order accurate least-square method, while in practice, the normal matrix of higher order least-square method is always ill-conditioned, even with the help of various weighting functions.

In this paper a new method is developed for the near wall treatment of block structured Cartesian mesh for the high Reynolds number flow simulation. In the near-body region, clouds of points are employed to resolve the boundary layer and most of the points are generated along one dimensional wall-normal lines. High order finite difference method is used to calculate the flow derivative along the wall-normal direction. To attain flexibility, least-square meshless method is used to approximate the flow derivative in the streamwise direction. Thus this method relives the burden of near wall mesh generation and also provides the opportunity of high order accuracy, at least in the wall-normal direction. This paper is organized as follows: in the second part the formulation of the hybrid method is introduced. The third part discusses the computational results of the current hybrid method. The last part concludes the paper and discusses future directions of research.

\section{Numerical methods}

\subsection{Governing equation and brief introduction of BCM}

The governing equation can be written in the Cartesian coordinate as

$$
\frac{\partial \mathbf{U}}{\partial t}+\frac{\partial \mathbf{F}}{\partial x}+\frac{\partial \mathbf{G}}{\partial y}=0
$$

in which $\mathbf{U}=[\rho, \rho u, \rho v, \rho E]^{T}$ denotes the conservative variable. $\mathbf{F}$ and $\mathbf{G}$ denote the convective fluxes and also include viscous fluxes for viscous cases. The detailed expressions for $\mathbf{F}$ and $\mathbf{G}$ can be found in textbook and will be omitted here for short.

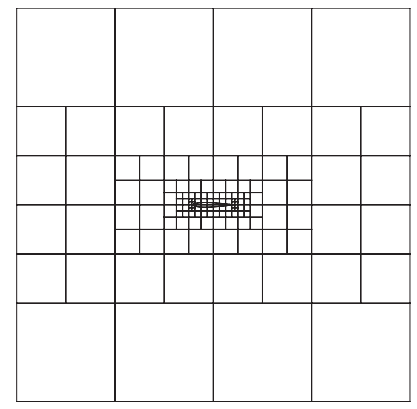

Fig. 1 BCM cubes in the computational domain

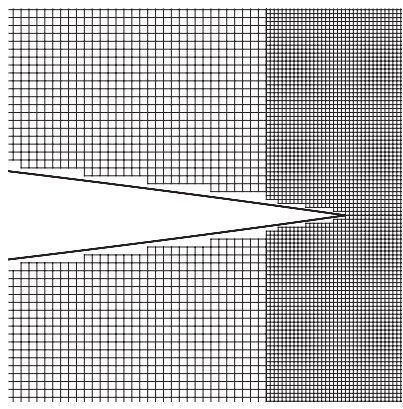

Fig. 2 Near-wall view of Figure 1

In the BCM method, the computational domain is meshed with block structured Cartesian mesh and every block is denoted as a cube, as demonstrated in Figure 1. Every cube has the 
same number of mesh points and the local near-wall view of Figure 1 is given in Figure 2, note that in this figure the points inside the solid are blanked. As Cartesian mesh is used and the mesh lines are not always aligned with the solid and wall treatment method is needed. Immersed boundary method ${ }^{(8)}$ and the least-square method can be used and in this paper the latter is interested. In the current work, for the off-wall region structured mesh, the third order upwind finite volume method is used to discretize the governing equation and Lagrange interpolation is used at the non-matching cube interfaces. The detailed implementation is described in Nakahashi et al. ${ }^{(3),(4)}$ and will not be repeated here.

\subsection{Hybrid finite difference and least-square method}

The meshless method uses cloud of supporting points to calculate the flow derivative, as shown in Figure 3. For point indexed 0, by assuming a linear distribution of any physical

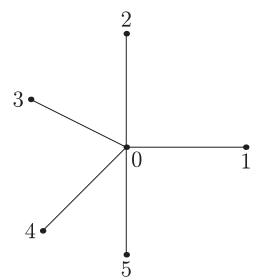

Fig. 3 Cloud of least-square meshless supporting points

variable $q, \nabla q$ can be obtained by solving the over-determined least-square system

$$
\min \sum_{i=1}^{n} w_{i}\left[q_{0}+\nabla q \cdot\left(\mathbf{r}_{i}-\mathbf{r}_{0}\right)-q_{i}\right]^{2}
$$

in which $w$ denotes the weight function and $\mathbf{r}$ denotes the position vector. Solving the above linear least-square problems yields the second order approximation of $\nabla q$

$$
\frac{\partial q}{\partial x}=\sum_{i=1}^{n} a_{i}\left(q_{i}-q_{0}\right), \quad \frac{\partial q}{\partial y}=\sum_{i=1}^{n} b_{i}\left(q_{i}-q_{0}\right)
$$

In the above equation, coefficients $a_{i}$ and $b_{i}$ are only related to the positions of the least-square supporting nodes. With the aid of Eq. 4, Eq. 2 can be semi-discretized as

$$
\frac{\partial \mathbf{U}}{\partial t}+\sum_{i=1}^{n}\left[\mathbf{F}_{i}, \mathbf{G}_{i}\right] \cdot\left[a_{i}, b_{i}\right]^{T}-\left[\mathbf{F}_{0}, \mathbf{G}_{0}\right] \cdot \sum_{i=1}^{n}\left[a_{i}, b_{i}\right]^{T}=0
$$

which is similar to that in the widely used finite volume method.

Suppose a cloud of points are generated to resolve the boundary layer and some points are located on the lines along the wall normal direction, as demonstrated in Figure 4. Thus we

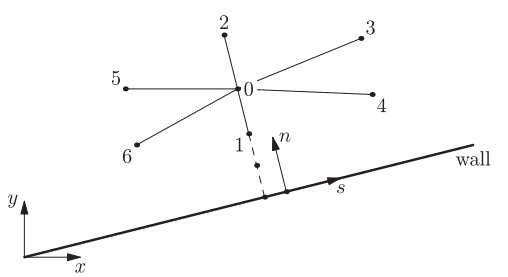

Fig. 4 Cloud of supporting points for the hybrid method

can setup a local coordinate system $(n, s)$ in which the new axis $n$ is perpendicular to the solid wall and the remaining axis $s$ is in the streamwise direction. With coordinate transformation, the governing equation in Eq. 2 can be transformed to the local coordinate system $(n, s)$ as

$$
\frac{\partial \mathbf{U}}{\partial t}+\frac{\partial\left(\mathbf{F} n_{x}+\mathbf{G} n_{y}\right)}{\partial n}+\frac{\partial\left(\mathbf{F} s_{x}+\mathbf{G} s_{y}\right)}{\partial s}=0
$$


The least-square problem in Eq. 3 is formed in the Cartesian coordinates and in the $(n, s)$ coordinate system a similar least-square problem can be expressed as

$$
\min \sum_{i=1}^{n} w_{i}\left[q_{0}+\nabla q \cdot\left(\mathbf{r}_{n s, i}-\mathbf{r}_{n s, 0}\right)-q_{i}\right]^{2}
$$

in which $\mathbf{r}_{n s}$ denotes the position vector in the new coordinate system. By solving this leastsquare problem we can have

$$
\frac{\partial q}{\partial n}=\sum_{i=1}^{n} a_{i}^{\prime}\left(q_{i}-q_{0}\right), \quad \frac{\partial q}{\partial s}=\sum_{i=1}^{n} b_{i}^{\prime}\left(q_{i}-q_{0}\right)
$$

With the expressions of $\partial / \partial n$ and $\partial / \partial s$ in Eq. 8, the governing equation in Eq. 6 can also be semi-discretized as

$$
\frac{\partial \mathbf{U}}{\partial t}+\sum_{i}\left(\mathbf{F}_{i}-\mathbf{F}_{0}\right)\left(a_{i}^{\prime} n_{x}+b_{i}^{\prime} s_{x}\right)+\sum_{i}\left(\mathbf{G}_{i}-\mathbf{G}_{0}\right)\left(a_{i}^{\prime} n_{y}+b_{i}^{\prime} s_{y}\right)=0
$$

If Eq. 9 is directly used, it can be easily proved for Cartesian mesh points this expression reverts to pure central difference and is unstable for hyperbolic problems. In this paper the upwind flux is used. The spatial residual in Eq. 9 can be re-formulated as

$$
\begin{aligned}
& \sum_{i}\left[\mathbf{F}_{i}, \mathbf{G}_{i}\right] \cdot\left[a_{i}^{\prime} n_{x}+b_{i}^{\prime} s_{x}, a_{i}^{\prime} n_{y}+b_{i}^{\prime} s_{y}\right]^{T} \\
& -\left[\mathbf{F}_{0}, \mathbf{G}_{0}\right] \cdot \sum_{i}\left[a_{i}^{\prime} n_{x}+b_{i}^{\prime} s_{x}, a_{i}^{\prime} n_{y}+b_{i}^{\prime} s_{y}\right]^{T}
\end{aligned}
$$

For the cloud of points in Figure 4, now the positions of the least-square supporting points are located at the mid-point of the edges connecting these points, as given in Figure 5. Also the coordinates of these new points, such as those indexed by $1^{\prime}$ and $2^{\prime}$ are used to solve the least-square problem in Eq.7 and to compute and least-square coefficients $a_{i}^{\prime}$ and $b_{i}^{\prime}$ in Eq. 8 . Denote $\mathbf{F}_{n, i}^{\prime}=\left[\mathbf{F}_{i}, \mathbf{G}_{i}\right] \cdot\left[a_{i}^{\prime} n_{x}+b_{i}^{\prime} s_{x}, a_{i}^{\prime} n_{y}+b_{i}^{\prime} s_{y}\right]^{T}$, in this paper $\mathbf{F}_{n, i}^{\prime}$ is computed using the

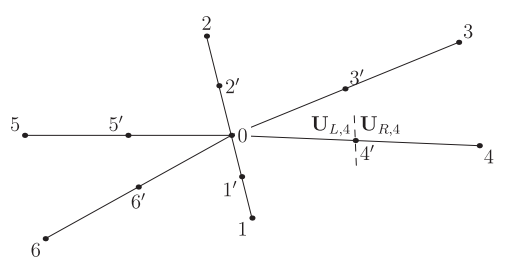

Fig. 5 Supporting nodes for the least-square based method using upwind flux

HLLC approximate Riemann solver ${ }^{(9)}$ and can be expressed as $\mathbf{F}_{n, i}^{\prime}=\mathbf{F}_{n, i}^{\prime}\left(\mathbf{U}_{L, i}, \mathbf{U}_{R, i}\right)$. For example, at point indexed by $4^{\prime}$, if $\mathbf{U}_{L, 4}=\mathbf{U}_{0}$ and $\mathbf{U}_{R, 4}=\mathbf{U}_{4}$, the spatial accuracy is only first order. For second order accuracy, the left and right state variables are calculated by

$\mathbf{U}_{L, 4}=\mathbf{U}_{0}+\nabla \mathbf{U}_{0} \cdot\left(\mathbf{r}_{4^{\prime}}-\mathbf{r}_{0}\right), \mathbf{U}_{R, 4}=\mathbf{U}_{4}+\nabla \mathbf{U}_{4} \cdot\left(\mathbf{r}_{4^{\prime}}-\mathbf{r}_{4}\right)$

In both Eq. 4 and Eq. 8, the accuracy is limited to second order. For high Reynolds number flow, the flow near the solid wall is highly anisotropic and there are large velocity gradients in the boundary layer, thus high order scheme for this area is preferable. The current idea is to use high accuracy algorithm in the wall-normal direction to resolve the large gradient in this direction, while second order least-square method is used in the streamwise direction for its superior flexibility. As given in Figure 4, several points, such as the points indexed by 0,1 and 2 , are positioned on the one dimensional line perpendicular to the solid wall. As a

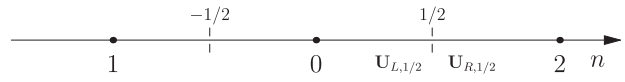

Fig. 6 1D problem in the wall-normal direction of Figure 4

result the wall normal direction falls into a purely one-dimensional problem and numbers of 
matured highly accurate algorithms are available. For the points located on the wall-normal lines, such as those indexed by 0,1 and 2 in figure 4, the flow derivative along the wall-normal direction $\partial / \partial n$ can be approximated by $1 \mathrm{D}$ high order schemes, such as the high order finite difference $^{(1)}$ or compact difference ${ }^{(2)}$. In the current method finite difference is used in the wall-normal direction. As the mesh points are always not equally distributed in this direction, one dimensional coordinate transformation is used for a set of equally spaced points. Denote $\mathbf{F}_{n}=\mathbf{F} n_{x}+\mathbf{G} n_{y}$, as given in Figure 6, the wall-normal part of the residual in Eq. 6 can be discretized as

$$
\frac{\partial \mathbf{F}_{n}}{\partial n}=\frac{\partial \mathbf{F}_{n}}{\partial \xi} \frac{\partial \xi}{\partial n}=\frac{\partial \xi}{\partial n}\left(\mathbf{F}_{n, 1 / 2}-\mathbf{F}_{n,-1 / 2}\right)
$$

In this paper, the numerical flux $\mathbf{F}_{n, 1 / 2}$ is computed using the HLLC Riemann solver ${ }^{(9)}$ and can be expressed as $\mathbf{F}_{n, 1 / 2}=\mathbf{F}_{n, 1 / 2}\left(\mathbf{U}_{L, 1 / 2}, \mathbf{U}_{R, 1 / 2}\right)$. The same as in the BCM solver, third order WENO reconstruction is used to compute $\mathbf{U}_{L, 1 / 2}$ and $\mathbf{U}_{R, 1 / 2}$.

Up-to-now, the high order scheme in the wall-normal direction and the second order least-square method can be combined to form the hybrid method, in that high order finite difference method is used to approximate the $\frac{\partial\left(\mathbf{F} n_{x}+\mathbf{G} n_{y}\right)}{\partial n}$ term in Eq. 6 and the second least-square method is used to approximate the $\frac{\partial\left(\mathbf{F} s_{x}+\mathbf{G} s_{y}\right)}{\partial s}$ term. In this case the governing equation in Eq. 6 can be semi-discretized using the hybrid method as

$$
\begin{aligned}
& \frac{\partial \mathbf{U}}{\partial t}+\frac{\partial \xi}{\partial n}\left(\mathbf{F}_{n, 1 / 2}-\mathbf{F}_{n,-1 / 2}\right)-\left[\mathbf{F}_{0}, \mathbf{G}_{0}\right] \cdot \sum_{i}\left[b_{i}^{\prime} s_{x}, b_{i}^{\prime} s_{y}\right]^{T} \\
& +\sum_{i}\left[\mathbf{F}_{i}, \mathbf{G}_{i}\right] \cdot\left[b_{i}^{\prime} s_{x}, b_{i}^{\prime} s_{y}\right]^{T}=0
\end{aligned}
$$

In calculating the terms in the second line of Eq. 13, the HLLC solver with second accuracy as demonstrated in Eq. 11 is used. For all the turbulent cases in this work, the Spalart-Allmaras turbulence model ${ }^{(10)}$ is used. The governing equation is discretized with the same method and will not be repeated here.

Compared to the off-wall BCM mesh, if second order least-square method is used, the elapsed time every near-wall point is about $80 \%$ larger. However, in the current hybrid method, as the wall-normal direction is discretized using the one-dimensional finite difference method and has similar CPU cost as the off-wall BCM part, thus the CPU cost overhead every point for the current hybrid method is reduced to $40 \% \sim 50 \%$. As only a small part of the mesh domain is solved using the hybrid method and the number of near-wall points is much smaller than that of the off-wall BCM mesh, compared to the computation without using the current wall-treatment method, for the test cases given in the paper, the overall cost overhead is in the range of $10 \% \sim 20 \%$.

\subsection{Implicit solution of the hybrid method}

As the current hybrid method is targeted at the wall treatment for high Reynolds number flows, the governing equation should be solved with method with which large CFL number is allowed for rapid convergence. In the current work, the semi-discretized system in Eq. 13 is solved with the implicit LU-SGS method ${ }^{(11)}$. Denoting $\mathbf{G}_{n, i}$ be the numerical approximation of $\left[\mathbf{F}_{i}, \mathbf{G}_{i}\right] \cdot\left[b_{i}^{\prime} s_{x}, b_{i}^{\prime} s_{y}\right]^{T}$ using the HLLC Riemann solver, then the Eq. 13 can be discretized in the implicit form as

$$
\frac{\delta \mathbf{U}}{\delta t}+\frac{\partial \xi}{\partial n}\left(\mathbf{F}_{n, 1 / 2}^{m+1}-\mathbf{F}_{n,-1 / 2}^{m+1}\right)+\sum_{i} \mathbf{G}_{n, i}^{m+1}-\left[\mathbf{F}_{0}^{m+1}, \mathbf{G}_{0}^{m+1}\right] \cdot \sum_{i}\left[b_{i}^{\prime} s_{x}, b_{i}^{\prime} s_{y}\right]^{T}=0(14)
$$

where the superscript $m+1$ denotes the number of iteration. The numerical fluxes in Eq. 14 can be expanded using Taylor series, for example, at interface 1/2,

$$
\begin{aligned}
\mathbf{F}_{n, 1 / 2}^{m+1} & =\mathbf{F}_{n, 1 / 2}^{m}+\left.\frac{\partial \mathbf{F}_{n}}{\partial \mathbf{U}}\right|_{L, 1 / 2} \delta \mathbf{U}_{L, 1 / 2}+\left.\frac{\partial \mathbf{F}_{n}}{\partial \mathbf{U}}\right|_{R, 1 / 2} \delta \mathbf{U}_{R, 1 / 2}+O\left(\delta \mathbf{U}^{2}\right) \\
& =\mathbf{F}_{n, 1 / 2}^{m}+\mathbf{A}_{L, 1 / 2} \delta \mathbf{U}_{L, 1 / 2}+\mathbf{A}_{R, 1 / 2} \delta \mathbf{U}_{R, 1 / 2}+O\left(\delta \mathbf{U}^{2}\right)
\end{aligned}
$$


Also $\mathbf{G}_{i}^{m+1}$ can be expanded as

$$
\begin{aligned}
\mathbf{G}_{n, i}^{m+1} & =\mathbf{G}_{n, i}^{m}+\left.\frac{\partial \mathbf{G}_{n}}{\partial \mathbf{U}}\right|_{L, i} \delta \mathbf{U}_{L, i}+\left.\frac{\partial \mathbf{G}_{n}}{\partial \mathbf{U}}\right|_{R, i} \delta \mathbf{U}_{R, i}+O\left(\delta \mathbf{U}^{2}\right) \\
& =\mathbf{G}_{n, i}^{m}+\mathbf{B}_{L, i} \delta \mathbf{U}_{L, i}+\mathbf{B}_{R, i} \delta \mathbf{U}_{R, i}+O\left(\delta \mathbf{U}^{2}\right)
\end{aligned}
$$

First order approximation is used to enhance the diagonal dominance property in deducing the left hand system, in that

$$
\begin{array}{ll}
\delta \mathbf{U}_{L, 1 / 2}=\delta \mathbf{U}_{0}, & \delta \mathbf{U}_{R, 1 / 2}=\delta \mathbf{U}_{2} \\
\delta \mathbf{U}_{L,-1 / 2}=\delta \mathbf{U}_{1}, & \delta \mathbf{U}_{R,-1 / 2}=\delta \mathbf{U}_{0} \\
\delta \mathbf{U}_{L, i}=\delta \mathbf{U}_{0}, & \delta \mathbf{U}_{R, i}=\delta \mathbf{U}_{i}
\end{array}
$$

Let

$$
\mathbf{C}=\frac{\partial\left[\mathbf{F}_{0}, \mathbf{G}_{0}\right] \cdot \sum_{i}\left[b_{i}^{\prime} s_{x}, b_{i}^{\prime} s_{y}\right]^{T}}{\partial \mathbf{U}},
$$

then the matrix form of Eq. 14 can be expressed as

$$
\begin{aligned}
& \left(\frac{\mathbf{I}}{\delta t}+\mathbf{A}_{L, 1 / 2}-\mathbf{A}_{R,-1 / 2}+\sum_{i} \mathbf{B}_{L, i}+\mathbf{C}\right) \delta \mathbf{U}_{0} \\
& +\mathbf{A}_{R, 1 / 2} \delta \mathbf{U}_{2}-\mathbf{A}_{L,-1 / 2} \delta \mathbf{U}_{1}+\sum_{i} \mathbf{B}_{R, i} \delta \mathbf{U}_{i}+R h s_{0}=0
\end{aligned}
$$

where Rhs is the right-hand-system. In this paper the Gauss-Seidel method with two successive forward and backward iterations are used to solve Eq. 19. In order to avoid the expensive matrix inversion, the matrix

$$
\frac{\mathbf{I}}{\delta t}+\mathbf{A}_{L, 1 / 2}-\mathbf{A}_{R,-1 / 2}+\sum_{i} \mathbf{B}_{L, i}+\mathbf{C}
$$

in Eq. 19 is replaced by its spectral radius to reduce the cost and enhance the diagonal dominance property for better stability.

\subsection{Generation of near wall points}

In existing work ${ }^{(6),(7),(12)}$ using the least-square method for the near wall treatment, a suit of near-wall mesh is first generated and then the least-square points are obtained by simply neglecting the topology information of the resulted mesh. These methods should be categorized as "semi-least-square" method, as the burden of near-wall mesh generation has not been relieved. Löhner and Onate ${ }^{(13)}$ proposed the advancing front method to obtain near isotropic points at fast-turnaround speed. The generated least-square points are suited for Euler inviscid computations and the generation of least-square points suited for viscous computation still remains an open question.

In the current hybrid method, a scatter of points is used in the near wall region to discretize the governing equation. In order to enable the finite difference method in the wallnormal direction, most of the points are located on the one dimensional lines normal to the solid surface. Also some points have to be generated for the smooth transition from the nearwall mesh points to the off-wall BCM mesh. The generation of the near-wall points is simply composed of two steps and is described as follows.

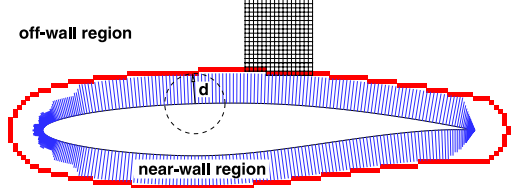

Fig. 7 Definition of near-wall region and the generation of one dimensional points

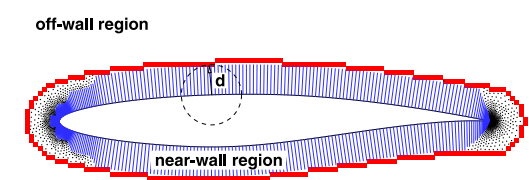

Fig. 8 Generation of least-square points for the smooth transition 
( 1 ) In the first step, the near-wall region to be discretized by the hybrid method is defined, as depicted in Figure 7. In the current method, the boundary layer region is fully covered by these points and thus the estimated boundary layer thickness in Eq. 1 can be used to estimate the depth of the near-wall region. As demonstrated in Figure 7, the inner boundary of the nearwall region is the solid wall and the outer boundary is positioned at the cell centers of the BCM mesh whose distance to the nearest wall approximately equal to $d$. In this manner the physical values at these cell centers can be directly exchanged between the near-wall mesh and the offwall BCM mesh without resorting to interpolations. Then the solid surface is discretized with properly distributed points. At every solid surface points, with the local wall-normal vector, one dimensional line can be generated and a set of points are located on this line to resolve the wall-normal direction of the boundary layer. For the smooth transition of mesh sizes between the near-wall mesh and the off-wall BCM mesh, in regions with large curvatures, such as the leading edge and the trailing edge, the generation of one dimensional points is ceased.

(2) At the end of the first step, most of the near-wall region is covered by points positioned on the one-dimensional lines. However, as the near-wall region the points are always anisotropic and in the off-wall region the points are isotropic, as given in Figure 7, there are resolution discrepancies and some additional points are needed for the smooth transition, as demonstrated in Figure 8. As these resolution discrepancies always take place far from the solid wall, so the method proposed by Löhner and Onate is used in this paper.

In the current work, the density of the near-wall cloud of points is determined automatically. As demonstrated in Figure 7, in the region where the solid has small radius of curvature, the streamwise density of the near-wall points is the same as the mesh size of the background BCM mesh (only one block is given in the picture for better vision). In regions the solid wall has large radius of curvature, the streamwise density is adapted to the local radius of curvature for better resolution of the near-wall flow features. In the wall-normal direction the first off-wall point is positioned to guarantee the $y^{+}$is approximately 1 and then the wall-normal direction cloud density is smoothly transitioned to the mesh size of the background BCM mesh.

With the above two steps, the near-wall points can be generated quickly without using external mesh generation software. In the first step the generation of one dimension points is simple and fast using only the local wall-normal information. Also a small number of points are generated in the second step, thus the overall elapsed time in the generation of near-wall points in the current work is negligible.

\section{Results and discussion}

\subsection{Accuracy test of the hybrid method}

In this test case, the accuracy of the proposed hybrid method for grids with curvature is tested. The lower and upper walls are defined as curves with constant radius. As given in Figure 9, the mesh points are generated using a mapping from the cylindrical coordinates $(r, \theta)$ to the Cartesian coordinates $(x, y)$. In this manner the points which satisfy $\theta=$ constant are

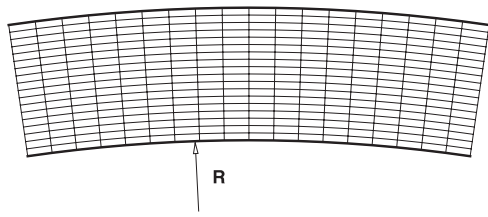

Fig. 9 Mesh used for the accuracy test of the proposed hybrid method

positioned on the wall-normal lines. A scalar field in the form of $f=\sin (5 \pi r)$ is assumed and the computed gradient $\nabla f$ is compared with the theoretical value.

Let $\delta r$ and $\delta \theta$ be the mesh spacings in the cylindrical coordinate system, then the curva- 
ture of the mesh proposed by Diskin and Thomas ${ }^{(14)}$ is defined in the form of

$$
\Gamma=R \frac{\delta \theta^{2}}{2 \delta r}
$$

As discussed by Diskin and Thomas ${ }^{(14)}$, for mesh with high curvature, the finite volume method, also the least-square method show obvious accuracy degradation. In the current work, the accuracy of the current hybrid method is assessed by increasing the curvature.
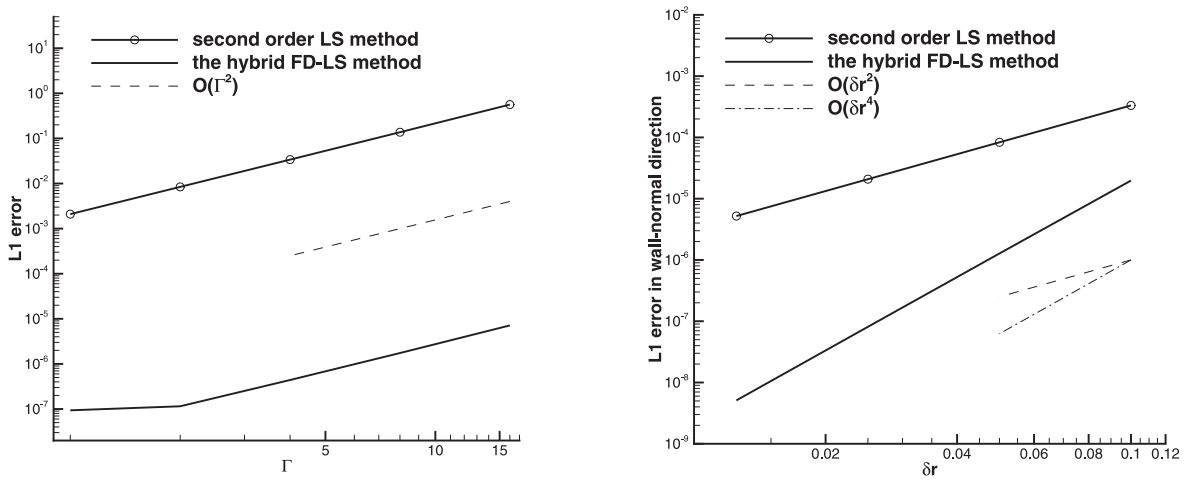

Fig. 10 Comparison of L1 norm errors of $\nabla f$ with Fig. 11 Comparison of L1 norm errors of wall-normal different $\Gamma$ part of $\nabla f$ with different mesh spacings

The L1 norm errors of $\nabla f$ are compared, as given in Figure 10. From this convergence study, the current hybrid method is globally second order, as second order least-square method is used in the streamwise direction. However, due to the use of higher order method in the wall-normal direction, the overall truncation error is reduced by several orders of magnitude, compared to the original second least-square method. Another set of convergence study with variable mesh spacing is conducted with constant curvature of $\Gamma=1$ to assess the accuracy in the wall-normal direction. As given in Figure 11, for the proposed hybrid method, the designed accuracy is attained and it is fourth order accurate in the wall-normal direction, compared to second order for the original least-square method. With the accuracy test, the improved accuracy of the proposed hybrid method is verified.

\subsection{Turbulent flow past the flat plate}

This test case is about the turbulent flow past the zero pressure gradient flat plate and is used to test the ability of the hybrid method to accurately resolve the boundary layer. For this test case, the off-wall region is meshed using the BCM method with 140 cubes and every cube is composed of $32 \times 32$ mesh points. 40 thousand points are used to resolve the nearwall region, including the points located on the one dimensional lines and the points used for the smooth transition. As in the current method the boundary layer is mostly discretized by the one dimensional points, thus an obvious virtue of current method is by specifying the one dimensional distribution of these points, the requirement of accurate capturing of the laminar sub-layer can be easily satisfied. In all the test cases in this work, the one dimensional distribution is specified to maintain $y^{+}$of the first off-wall point is less than 1 .

For the flat plate, the freestream Mach number is $M_{\infty}=0.2$ and the Reynolds number based on the length of the plate is $R e=5 \times 10^{6}$. With this set of freestream conditions, there are publicly available numerical results using the NASA CFL3D code and the NASA FUN3D code $^{(15)}$. At the streamwise position with the momentum thickness based Reynolds number $R e_{\tau}=10000$, the velocity profile is extracted and the distribution of streamwise velocity against the wall-normal coordinate is compared with the theoretical Coles velocity profile, as given in Figure 12.

The computed wall skin friction coefficients are also compared with the approximated 


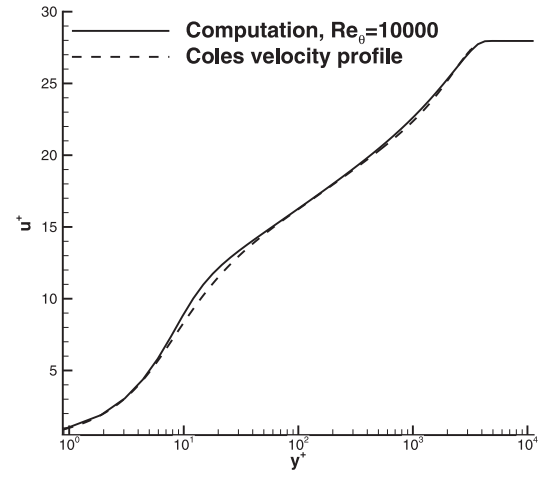

Fig. 12 Comparison of the dimensionless velocity profile with the Coles velocity profile

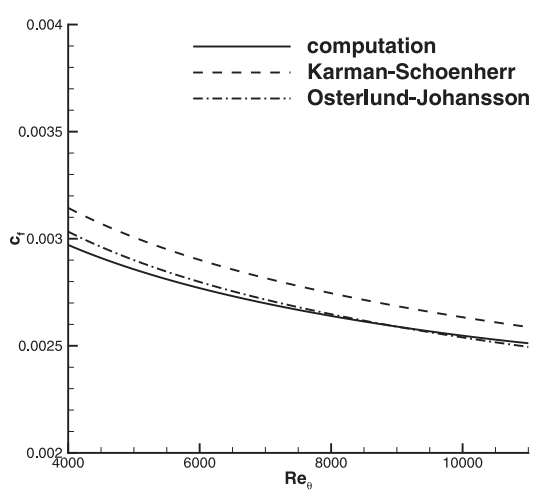

Fig. 13 Comparison of skin friction coefficients with various approximated relations

Karman-Schoenherr relation in which

$$
c_{f}=\left(17.08\left(\log _{10} R e_{\theta}\right)^{2}+25.11\left(\log _{10} R e_{\theta}\right)+6.012\right)^{-1}
$$

and also the much recent Osterlund-Johansson relation ${ }^{(16)}$ in which

$$
c_{f}=2\left(\frac{1}{0.384} \log R e_{\theta}+4.08\right)^{-2}
$$

The computed results agree well with the Osterlund-Johansson for $R e_{\theta}$ in the range between 4000 and 11000, as given in Figure 13.

\subsection{Turbulent flow past the NACA-0012 airfoil}

This test case is about the subsonic flow past the NACA-0012 airfoil. Again in this case the boundary layer is fully covered by the one dimensional distributed points. As the trailing edge of the airfoil is a singular point, thus currently in the region near the singular point, scattered points are generated using the method proposed by Löhner and Onate and the governing equation is solved purely using the second least-square method. This may result in a bit accuracy reduction near the trailing edge and according to the numerical result this effect is minor and negligible.

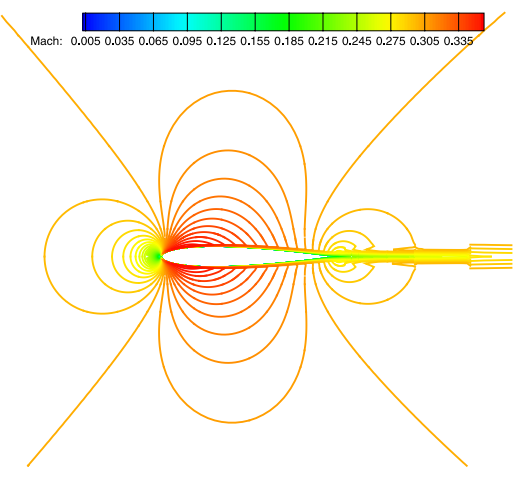

Fig. 14 Computed Mach number for the NACA-0012 test case with zero angle of attack

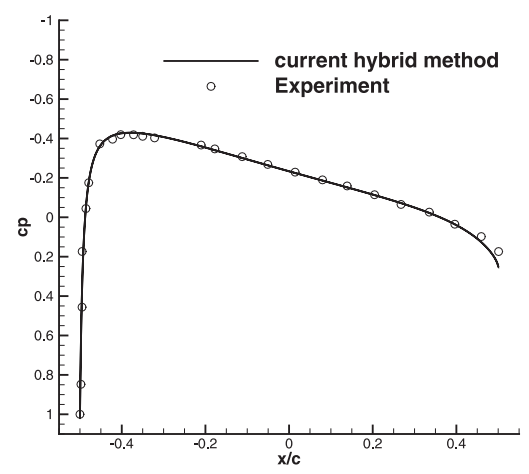

Fig. 15 Comparison of $\mathrm{cp}$ with the experiment value, zero angle of attack

The Reynolds number based on the chord length is $R e=6 \times 10^{6}$. For the NACA-0012 test case, two sets of numerical experiments are conducted. In the first set the angle of attack is zero. The computed Mach number distribution is given in Figure 14. As NACA-0012 is a symmetrical airfoil, at zero angle of attack the upper and lower surfaces pressure coefficients are also symmetrical. The comparison of computed upper surface pressure coefficients are 


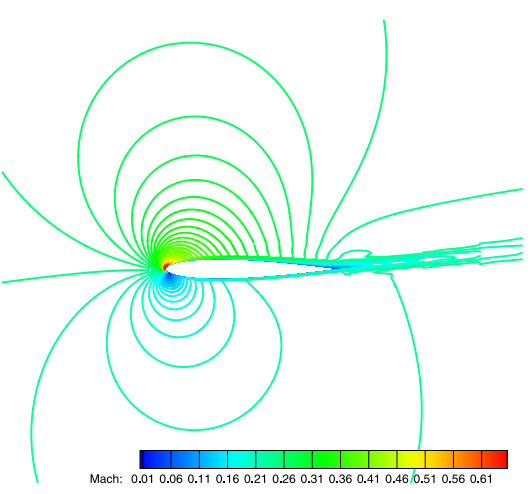

Fig. 16 Computed Mach number for the NACA-0012 test case, 10 degrees angle of attack

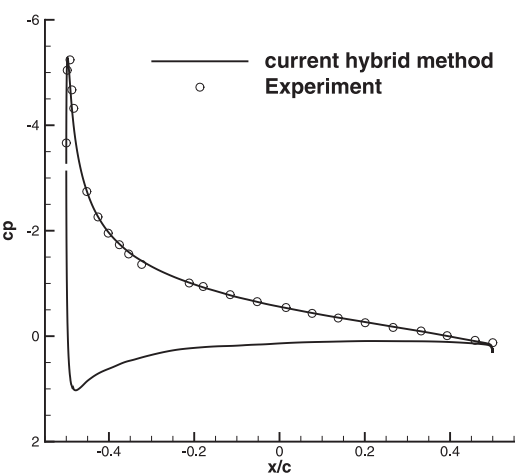

Fig. 17 Comparison of $\mathrm{cp}$ with the experiment value, 10 degrees angle of attack

compared with the experiment values ${ }^{(17)}$, as demonstrate in Figure 15. From the comparison, the numerical results using the hybrid method agree well with the experimental values.

In the second set the angle of attack is increased to 10 degrees. The computed Mach number distribution is given in Figure 16. Note that with 10 degrees angle of attack, the flow near the trailing edge tends separation and in this case the numerical results of the hybrid method again agree well with the experiment, as compared in Figure 17.

\subsection{Turbulent flow past the 30P-30N multi-element airfoil}

The fourth test case is an example of the turbulent flow past the 30P-30N multi-element airfoil. In this case the geometry is much complex and there are several singular points of the geometry, such as the convex points at the trailing edges and the concave point. Similar as in the NACA-0012 test case, near the singular points the computational domain is resolved using the second order least-square method. The generated one dimensional lines and leastsquare points in the leading edge area and also the concave area of the main airfoil are given in Figure 18. In the near-wall region 43 thousand points are used for the discretization of boundary layer and in the off-wall region 229 cubes are generated and every cube is composed of $32 \times 32$ mesh points.

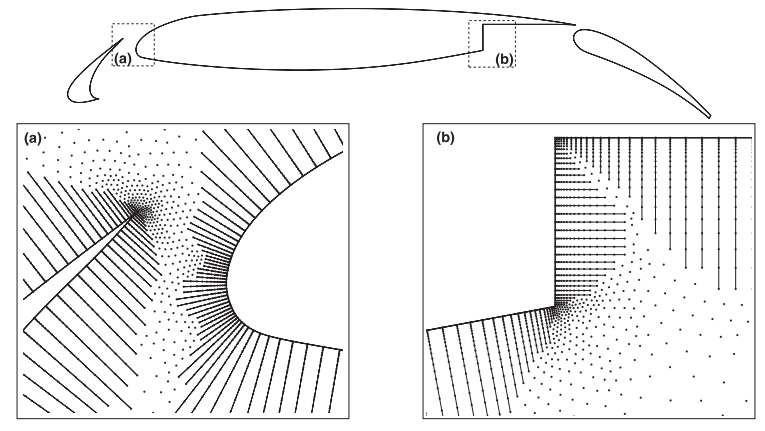

Fig. 18 Computed Mach number distribution for the 30P-30N test case

For the current $30 \mathrm{P}-30 \mathrm{~N}$ case, the freestream Mach number is $M_{\infty}=0.2$ and the Reynolds number is $R e=9 \times 10^{6}$. The computed Mach number distribution is given in Figure 19 . From the Mach number distribution, the wakes of the airfoil elements and the interactions of upstream wake with downstream boundary layer are clearly captured.

The comparison of computed surface pressure coefficients with experiment are given in Figure 20. From the comparison, the current numerical results are in general agreement with the experiment, except near the upper surfaces of the slat and the flap where the computed values are higher than the experiment. Computation with refined mesh in these areas has been conducted and it results in minor improvement. Near the upper surface of the slat the 


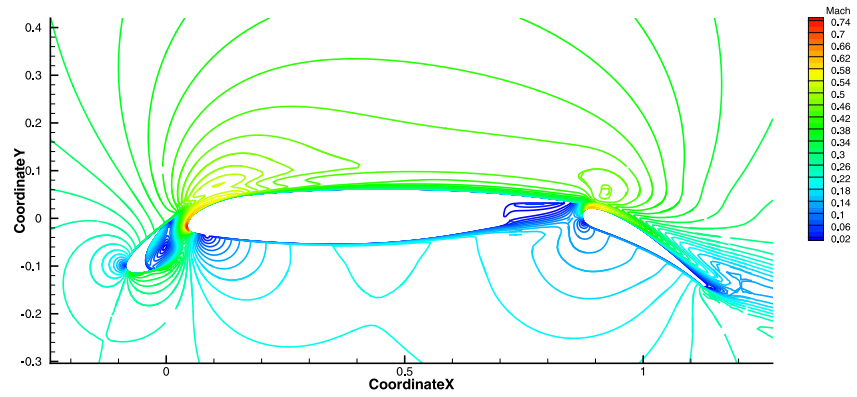

Fig. 19 Computed Mach number distribution for the 30P-30N test case

flow tends to separation and near the upper surface of the flap the flow is complex due to the interaction of the upstream wake and the boundary layer. Thus the difference is believed to be caused by the turbulence model and in future the Menter-SST turbulence model will be tested.

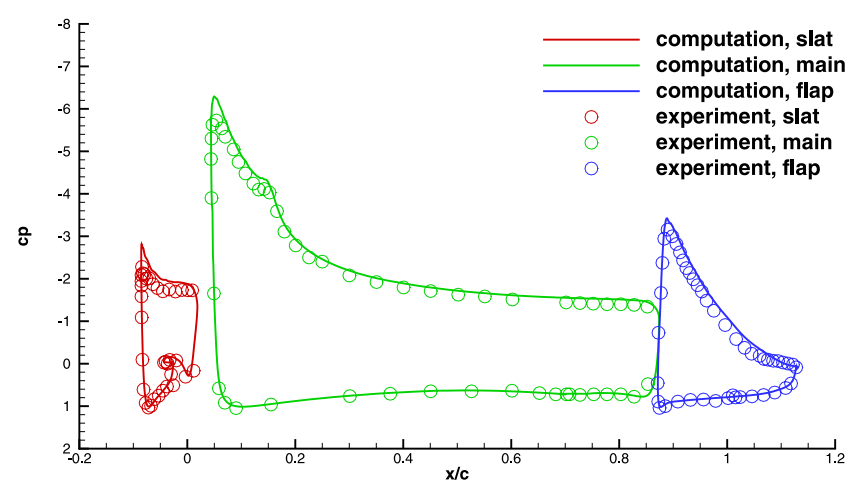

Fig. 20 Comparison of computed $\mathrm{cp}$ with the experiment for the $30 \mathrm{P}-30 \mathrm{~N}$ test case

\section{Conclusions}

In this work an efficient hybrid method aimed at the near-wall treatment of BCM method for high Reynolds number flow simulation is developed. The current method is a hybrid of high order finite difference method and second order least-square method. In the wall-normal direction high order finite difference is used to accurately resolve the boundary layer and at the same time second order least-square method is used in the streamwise direction. The proposed hybrid method combines the improved accuracy of high order finite difference and the flexibility of least-square method. A simple method is also proposed to generate the nearwall points for the hybrid method. Several numerical examples are conducted with the hybrid method and the accuracy is validated.

In the current work the hybrid method employs upwind based finite difference in the wallnormal direction. For high-fidelity simulations, such as Computational Aeroacoustics and Direct Numerical Simulation, compact finite difference is always used and it can be directly incorporated into the current methodology. Also the extension of the current method to three dimensional problem is of interest. These issues are topics of future research.

\section{Acknowledgements}

This work is supported by JSPS KAKENHI (21226018). Comments by anonymous reviewers are appreciated.

\section{References}

( 1 ) Guang Shan Jiang and Chi Wang Shu, Efficient implementation of Weighted ENO schemes, J. Comput. Phys., Vol.126 No.2 (1996), pp.202-228. 
( 2 ) Lele. S. K., Compact finite difference schemes with spectral-like resolution, J. Comput. Phys., Vol.103 No.1 (1992), pp.16-42.

( 3 ) Kazuhiro Nakahashi, High density mesh flow computations with Pre-/Post-data compressions, AIAA-2005-4876, 2005.

(4) Kazuhiro Nakahashi, Aya Kitoh, Yuta Sakurai and Matthias Meinke, Three dimensional flow computations around an airfoil by Building-Cube Method, AIAA-20061104, 2006.

( 5 ) Takashi Ishida, Soshi Kawai and Kazuhiro Nakahashi, A high resolution method for flow simulations with Cartesian mesh method, AIAA-2011-1296, 2011.

( 6 ) N. Munikrishna, N. Balakrishnan, Turbulent flow computations on a hybrid cartesian point distribution using meshless solver LSFD-U, Computers and Fluids, Vol.40 No.1 (2011), pp.118-138.

( 7 ) A. Jahangirian, M.Y. Hashemi, Adaptive Cartesian grid with mesh-less zones for compressible flow calculations, Computers and Fluids, Vol.54 No.2 (2012), pp.10-17.

( 8 ) Rajat Mittal and Gianluca Iaccarino. Immersed boundary methods. Annual Review of Fluid Mechanics, Vol.37 No.1 (2005), pp.239-261.

( 9 ) P. Batten, M. A. Leschziner and U. C. Goldberg, Average-state Jacobians and implicit method for compressible viscous and turbulent flows, J. Comput. Phys., Vol.137 No.1 (1997), pp.38-78.

(10) Spalart. P, Allmaras. S. A., A one equation turbulence model for aerodynamics flows, AIAA-1992-0439, 1992.

(11) Jameson, A., Yoon, S., Lower-upper implicit schemes with multiple grids for the Euler equations, AIAA Journal, Vol.25 No.7 (1987), pp.929-935.

(12) Katz, A., Jameson, A., Edge-based meshless methods for compressible flow simulations, AIAA-2008-699, 2008.

(13) Löhner, R., Onate, E., An advancing front point generation technique, Commun. Numer. Meth. Engng, Vol.14 No.1 (1998), pp.1097-1108.

(14) Boris Diskin, James L. Thomas, Comparison of node-centered and cell-centered unstructured finite volume discretizations: inviscid fluxes, AIAA Journal, Vol.49 No.4 (2011), pp.836-854.

(15) http://turbmodels. larc.nasa.gov

(16) Jens M. Osterlund, Arne V. Johansson, Hassan M. Nagib, Michael H. Hites, A note on the overlap region in turbulent boundary layers, Physics of Fluids, Vol.12 No.1 (2000), pp.1-4.

(17) Ladson, C. L., Effects of independent variation of Mach and Reynolds numbers on the low-speed aerodynamic characteristics of the NACA 0012 airfoil section, NASA-TM4074, 1988. 